\title{
Intelligent Charging Query System of Electric Vehicles Based on Internet of Things
}

\author{
Qinshan Han and Jialiang He* \\ College of Information and Communication Engineering, Dalian Nationalities University, China \\ ${ }^{*}$ Corresponding author
}

\begin{abstract}
The key technologies of Internet of Things includes RFID technology, sensor network and detection technology, intelligent technology, it has been applied in various areas and plays a vital part in normal life. This paper introduces the concept of electric vehicle charging inquiry system, operating mode, main technology and project value.
\end{abstract}

\section{Keywords-internet of things; charging query; electric vehicles}

\section{INTRODUCTION}

IoT has been used in many domains of traffic and logistics[1-5]. With the development of the economy, the environmental pollution becomes more and more serious and the automobile industry, as the main source of pollution, has gained more attention from the government and the public, meanwhile, the electric automobile industry is now facing a new opportunity. It has gradually been the high ground with considerable prospects for which all countries are competing to fight. However, the hardware facilities matched haven't been perfected on time and the difficulties in charging have restrained the pace of the electric automobile market and severely affected its popularization and growth. Therefore, to truly realize the application of electric automobile at the road traffic, the most urgent problem that needs to be solved is the charging problem.

To help solve the charging problem, we have developed the inquiring system for charging the electric automobile on the basis of the Internet of Things, hoping that it can help the users to utterly solve the problem that they can't timely and rapidly find the charging station nearby when the energy isn't sufficient wherever in the city or remote places. Besides, it also offers feasibility study for the rapid inquiry for charging the electric automobile into the Internet of Things.

Comparing with the detection model of the current charging station for electric automobile, the features of this project are as follows:

\section{A. Utilize Sensor Group and Network Server}

This project takes advantage of the mutual transmission of information among the sensors, network server and the intelligent terminal equipment so as to rapidly and accurately give feedbacks of the relevant information of the charging station nearby to the car owners in real time. It enables the car owners to get real-time information accurately so that they can either choose the route themselves or follow the best way which has been processed by the system.

\section{B. Avoid Waiting in Line for Charging Stations}

This system gets rid of the situation that the car owners have to wait at some charging station while some others are unoccupied. Through the sharing between the network service station and the real-time data, the system has improved the efficiency and abilities of processing both the information and data which enables the users to flexibly choose the spare charging station at any time according to their real-time status. In addition, it also saves the time for the car owners as well as avoids the troubles unwanted.

\section{Search Charging Station Nearby}

It can ascertain the specific position of the charging station nearby and the service condition of relevant chargers. The features are as follows: wide application, high utilization, fast speed, low cost, high accuracy, less affected by the environment, time and energy-saving, simple and convenient usage, flexible and reliable system, safe and durable equipment and clear target.

\section{THE FunCtions OF THE PROJECTS}

This system mainly consists of four modules: inquiry of real-time information of the charging station, auto match charging station, freely choosing charging station and navigation. Through the built-in maps and the positioning service, it promptly helps the users learn about the specific information of the charging station nearby and obtains its position and the number of spare charger. It deals with the problem that the charging station being left aside which is absolutely a waste of resources. In addition, it helps the users to find the charging station nearby shortly when their cars need to be charged.

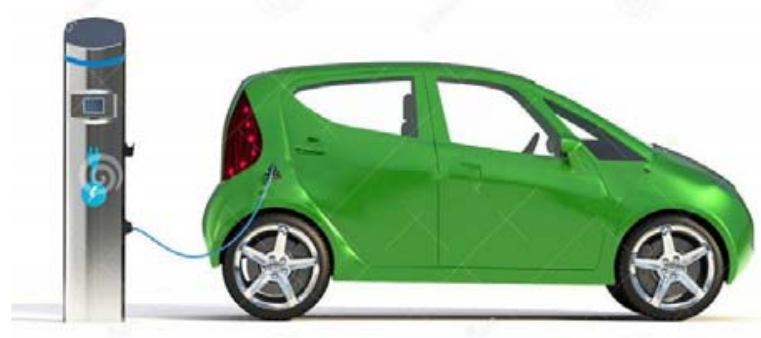

FIGURE I. SIMULATION FOR CHARGING ELECTRIC ARTOMOBILE 
Hereinto, the charging inquiry of the real-time information of the charging station is mainly completed by the real-time interaction between the system and the hardware facilities and it will regularly update the specific information of the charging station; system auto match is implemented by the safest routine shown which is the analyzed result of the information sent from the sensor group through relevant algorithm; free choosing refers to that the users can arbitrarily choose certain charging station and the system will show them the route to the destination; the voice navigation mainly helps the users to rapidly and effectively find the charging station and avoid the occurrence of missing the best charging time due to the unfamiliarity of local traffic and the misreading of the maps. Besides, it also gets rid of the troubles which resulted from paying attention to the maps.

\section{SYSTEM IMPLEMENTATION}

Based on the mutual transmission of information among the sensor group, microcontroller, WIFI network, network server and the intelligent terminal devices, this project reaches the goal of rapidly and accurately giving feedbacks of the relevant information of the charging station nearby to the electric car owners in real time.

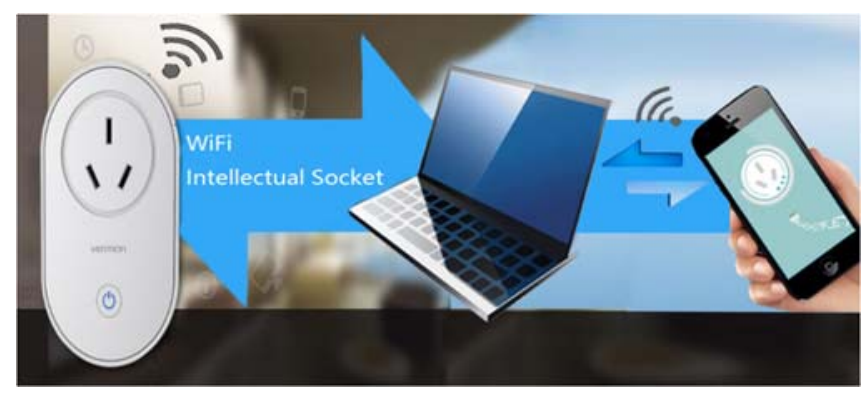

FIGURE II. SYSTEM STRUCTURE

Firstly, it realizes the connection between the sensor group and the charging device in the station. The charging system should collect and process the power parameters in the charging device and timely report back to both the users and the charging station the state of each device in which a connection system between the sensor group and the charging device needs to be established to complete the data transmission. See the figure below:

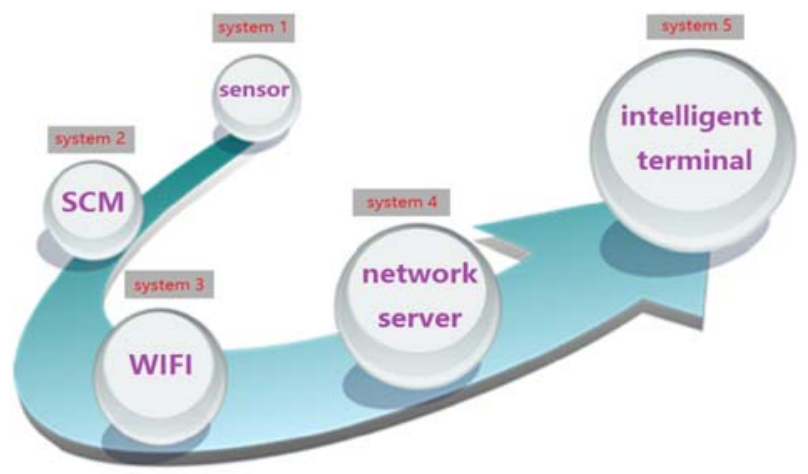

FIGURE III. THE PROCESS OF THE SYSTEM'S COLLECTION OF INFORMATION AND ITS TRANSMISSION

Secondly, the sensor group collects relevant data information in the charging device and delivers it to the network server through microcontroller and WIFI network. Meanwhile, it will position all charging station in the system and send their locations to the network server. The charging system will analyze and process these data and find the closest charging device available and show relevant information of the charging stations around. After the user chooses the auto selection, it will generate a best routine and then the network server will give feedback to the intelligent terminals of the processed information. So the user can see the usage of the charging station and the locations of those available as well as the suggested routes.

The core module of this system is the transmission process of the information among the sensor, microcontroller and the WIFI. It's the function that determines whether the equipment can communicate and complete the prospective of the system.

The final goal of the charging system is to effectively and accurately transmit the information among the sensor group, microcontroller, WIFI, website server, intelligent terminal and the users so as to fast and effectively deal with the practical problem of providing the electric automobile up to coming with cheap and environmentally-protective green power and promote the development of the electric automobile industry.

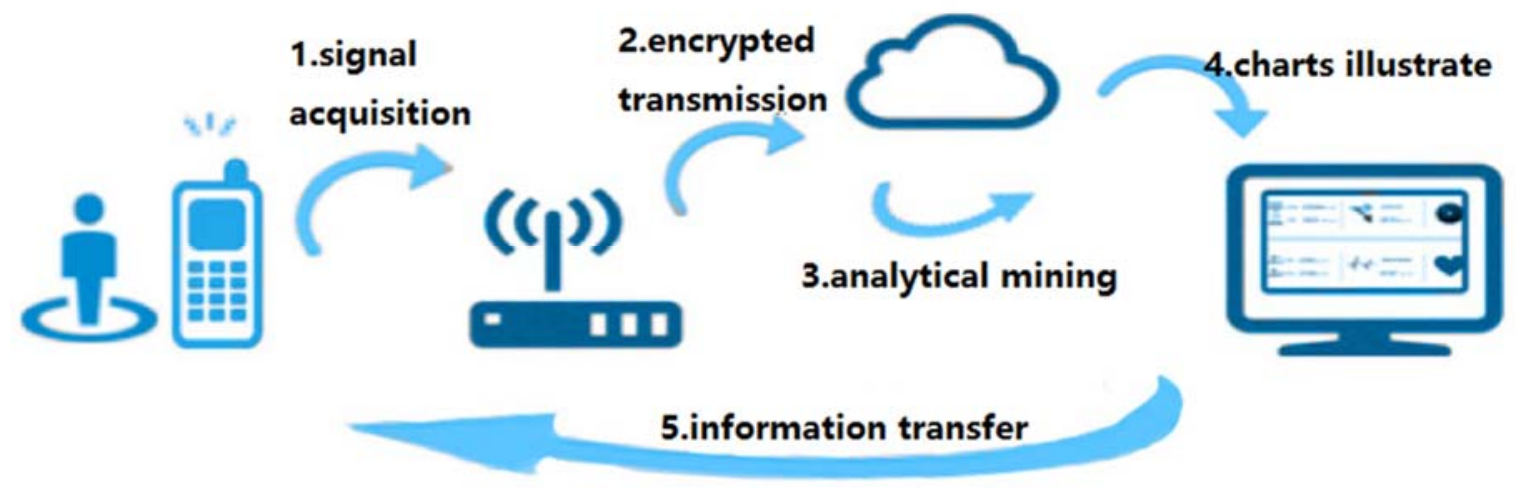

FIGURE IV. SIMULATED PROCESS OF THE SYSTEM OPERATION 
With the features of wide application, high utilization, fast speed and high accuracy, this project can ascertain the specific location of the charging station around the electric automobiles and the usage of the chargers as well as fast and effectively deal with the practical problem of providing the electric automobile up to coming with cheap and environmentally-protective green power.

\section{CONCLUSIONS}

On the basis of the national economy, people's living standard and the significant demand of the environmental protection for the internet of things as well as the data transmission among the sensor group, network service station and the intelligent terminals, it timely delivers the information to the electric car owners. the cooperation with application software, like AliPay can not only provide the user more convenient and favorable ways of payment, but also accelerate the promotion of the product and enlarge the user's group. In addition, it can improve the usage of the limited charger now and make them play their best role, meanwhile it also pushes the development of the electric automobile market and accelerates the relief the environmental pollution brought by the gas emission of the traditional cars through providing more and more convenience to the application of the electric automobiles.

\section{ACKNOWLEDGEMENTS}

This work was supported by "National Undergraduate Training Programs for Innovation and Entrepreneurship of Dalian Nationalities University”.

\section{REFERENCES}

[1] Feng Haiyang, He Jialiang, Zhang Lijun, Meng Fangang, An Application of RFID Technology in Traffic Congestion Management[C]. Applied Mechanics and Materials, 2014, vol.513-517, pp.3954-3957.

[2] Shen Peng, He Jialiang, Feng Haiyang, A Solution of Vehicle Emission Inspection Using RFID Technology[C]. 2014 International Conference on Vehicle, Mechatronics and Information Technologies II. Applied Mechanics and Materials, 2014, vols.543-547, pp.946- 949.

[3] Wang Huan, He Jialiang, Pan Yu. A Solution Framework in Traffic Congestion Management Using RFID Technology[C]. International Industrial Informatics and Computer Engineering Conference (IIICEC 2015). 2015, pp.1098-1101. Atlantis Press.

[4] He Jialiang, Shen Peng, Xu Zhiqiang. Inspection Solution of Unlawfully Modified Vehicle by Sensor-RFID Technology[J]. Sensors and Transducers Journal. 2013, vol.25, pp.197-202.

[5] He Jialiang, Xu Zhiqiang. Authentication and Search Mechanism for Diffusing RFID-Sensor Networks[J]. International Journal of Sensor Networks. 2013, vol.14, no.4, pp. 211-217. 